\title{
Subcortical dementia
}

\section{Distinguishing it from cortical dementia may be worthwhile}

Though subcortical dementia was first described in $1912,{ }^{1}$ the term was not used until the early 1970 s. $^{2}$ Subcortical structures include the basal ganglia, diencephalon (thalamus and hypothalamus), mesencephalon (midbrain), and cerebellum. They have a role in arousal, attention, mood, motivation, memory, abstraction, and visuospatial skills. Subcortical nuclei are affected in Parkinson's disease, progressive supranuclear palsy, Huntingdon's chorea, hydrocephalus, and the AIDS dementia complex. Additionally, depression may cause subcortical dementia. ${ }^{3}$ By contrast, Alzheimer's disease is held to be the classic example of a cortical dementia.

Subcortical dementia is noted for a special type of cognitive impairment, characterised by marked psychosocial incompetence associated with minimal memory loss and, as a rule, absence of aphasia, apraxia, or agnosia. Characteristic symptoms include forgetfulness, slowing of thought processes, mild intellectual impairment, apathy, inertia, depression (sometimes with irritability), and the inability to manipulate knowledge. ${ }^{24}$ Difficulties in problem solving and abnormalities of judgment and insight may occur. ${ }^{5}$

Conceptually, cortical abilities can be categorised as subserving instrumental functions (language, perception, memory, and calculation) and subcortical abilities as subserving fundamental functions (arousal, mood, and motivation). Cortical dysfunction leads to aphasia, agnosia, amnesia, and acalculia; subcortical dysfunction slows information processing and adversely affects memory, cognition, mood, and motivation. ${ }^{6}$ Memory impairment is characterised by a greater deficit of spontaneous recall than of encoding and storage of new material, which is characteristic of the cortical dementias. ${ }^{7}$ As a result, memory assessed by clues and recognition tests is usually superior to free recall. Articulation and verbal fluency may be impaired, but language is largely spared in the subcortical dementias. ${ }^{6}$

About a tenth of patients with Parkinson's disease develop cortical dementia, and two thirds may be affected by frontal subcortical dysfunction. ${ }^{8}$ Early in the course of the disease many patients show subtle deficits of the subcortical type, which are attributed to the loss of striatal and cortical dopaminergic innervation, secondary to cell loss in the substantia nigra. Multiple sclerosis almost invariably affects the subcortical white matter, and the pattern of cognitive impairment (which occurs in $30-70 \%$ of patients) suggests a subcortical dementia. ${ }^{10}$

The subtle inability to plan and organise behaviour may result from minor involvement of the frontal lobes. The cerebral cortex projects to the neostriatum, which in turn connects to the thalamus, which feeds back to the cortex, completing a loop. Thus, the prefrontal areas of the cortex are closely connected to the subcortical nuclei, and dysfunction in the nuclei would theoretically deactivate an otherwise normal cortex; this could explain the predominance of frontal features in subcortical dementia.

Several important transmitters, including dopamine and noradrenaline, have their cells of origin in subcortical nuclei and project to diencephalic and telencephalic structures via subcortical tracts. The basal ganglia and substantia nigra also receive extensive projections from the limbic system, and disruption of these pathways may partly account for the disturbances in mood and motivation associated with subcortical dementias. Surgical ablation of the dorsomedial thalamic nucleus results in complex changes in drive, problem solving ability, personality, and feeling. The medial and rostral parts of the thalamus subserve recent memory function, and a normally functioning circuit from the hippocampus via thalamus and limbic system to the cortex is necessary for establishing a recent memory trace.

A synthetic analogue of the analgesic meperidine, 1-methyl4-phenyl-1,2,3,6-tetrahydropyridine (MPTP), selectively destroys dopaminergic neurones in the substantia nigra, and assessment of patients given the drug shows abnormalities of attention and cognition consistent with subcortical dementia." Investigations using positron emission tomography have shown decreased metabolism in the striatum with normal cerebral metabolism in patients with Huntingdon's disease. ${ }^{12}$

The concept of subcortical dementia ${ }^{13}$ has been criticised by some investigators, ${ }^{2}$ who view it as a milder form of dementia and argue that not all dementias can be neatly classified as either cortical or subcortical. For example, in diffuse Lewy body disease (which may account for up to one fifth of cases previously categorised as Alzheimer's disease) cortical atrophy may occur with neuronal loss in several subcortical structures. It is unsurprising that frontal lobe syndromes and subcortical dementias are similar, given their anatomical connections. Subjects with subcortical symptoms may show classic cortical changes, both clinically and neuropathologically, and vice versa.

Increasingly sophisticated investigations (especially functional imaging) may show two broad diagnostic categories of cortical and subcortical dementia or perhaps that each 
disease has a characteristic picture of impairment, with a small number of shared features. ${ }^{14}$ At present there are no simple bedside tests to assess speed of cognition in patients with subcortical dementia, though more subtle tests of language, memory, problem solving, and naming objects represented by pictures may help differentiate it from the cortical variety. ${ }^{15}$

Distinguishing cortical from subcortical dementia may have some therapeutic value as states of subcortical dementia (in addition to parkinsonism) may be amenable to treatment with dopamine agonists and levodopa. Clinically, most treatable dementias exhibit a subcortical pattern, whereas Alzheimer's disease remains incurable. ${ }^{16}$ Better awareness of the cognitive deficits associated with particular dementias will hopefully lead to better management.

FRANCIS J DUNNE Consultant psychiatrist

Alzheimer's Disease Unit,

Warley Hospital,

Brentwood CM14 5HQ
1 Wilson SAK. Progressive lenticular degeneration: a familial nervous disease associated with cirnosis of the liver. Brain 1912;34:295-509.

2 Albert ML, Feldman RG, Willis AL. The "subcortical dementia" of progressive supranuclear palsy. I Neurol Neurosurg Psychiatry 1974;37:121-30.

3 Caine ED. Pseudodementia. Arch Gen Psychiatry 1981;38:1359-64.

4 Huber SJ, Shuttleworth EC. Neuropsychological assessment of subcortical dementia. In: Cummings JL, ed. Subcortical dementia. New York: Oxford University Press, 1990:71-86.

5 McHugh PR, Folstein MF. Psychiatric syndromes of Huntingdon's chorea: a clinical and phenomenologic study. In: Benson DF, Blumer D, eds. Aspects of neurologic disease. New York: Grune and Stratton, 1975:267-86.

6 Cummings JL. Subcortical dementia: neurophysiology, neuropsychiatry, and pathophysiology. Br F Psychiatry 1986;149:682-97.

Tweedy JR, Langer KG, McDowqell FH. The effect of semantic relations on the memory deficit associated with Parkinson's disease. 7 Clin Neuropsychol 1982;4:235-47.

Baldwin RC, Byrne EJ. Psychiatric aspects of Parkinson's disease. BM 1 1989;299:3-4

9 Baldwin RC, Byrne Ej. Psychiatric aspects of Parkinson's disease. BMf 1989;299:3-4.

10 Ron MA. Multiple sclerosis: psychiatric and psychometric abnormalities. If Psychosom Res 1986;30:3-11.

1 Ballard PA, Tetrad JW, Langston W. Permanent human parkinsonism due to 1-methyl-4-phenyl1,2,3,6-tetrahydropyridine (MPTP): seven cases. Neurology 1985;35:949-56.

12 Kuhl DE, Phelps ME, Markham CH, Metter EJ, Riege WH, Winter J. Cerebral metabolism and atrophy in Huntingdon's disease determined by 18 FDG and computed tomographic scan. Am Neurol 1982;15:411-24.

13 Mayeux R, Stern Y, Rosen J, Benson DF. Is "subcortical dementia" a recognizable clinical entity? Ann Neurol 1983;14:278-83.

4 Brown RG, Marsden CD. Subcortical dementia: the neuropsychological evidence. Neuroscience 1988;25:363-87.

15 Cummings JL Benson F. Subcortical dementia: review of an emerging concept. Arch Neurol 1984;41:874-79.

16 Hodges J. Subcortical dementia. Alzheimer's Review 1992;2:41-5.

\section{Sex, pregnancy, hormones, and melanoma}

\section{Exogenous oestrogens do not seem to have a role in causation}

The possible role of exogenous oestrogen in the aetiology of melanoma has aroused considerable controversy since the late 1970s when a study from the California Kaiser Permanente Health Plan suggested that oral contraceptives increased the risk of developing melanoma. ${ }^{1}$ Despite at least 11 case control studies since then the controversy persists, with most reports concentrating on the role of oral contraceptives rather than hormone replacement therapy. ${ }^{2}$ The main reason for the continuing debate is the difficulty in designing a study that takes into account important variables such as duration of oral contraceptive use, histological subtype, and aetiological factors such as sunlight. Although a link between oral contraceptive use and superficial spreading melanoma has been suggested, overall results do not suggest a substantial effect of oral contraceptive use on the aetiology of melanoma. ${ }^{23}$

Women with melanoma have a significantly better prognosis than men, with five year survival of $77 \%$ and $64 \%$ respectively. ${ }^{4}$ Several factors account for this: most women present with lesions on the legs, which carry a more favourable prognosis, while men present mainly with lesions on the trunk. ${ }^{4}$ Furthermore, on presentation the average tumour thickness is significantly less in women, ${ }^{5}$ although this may reflect the tendency of women to seek medical attention earlier. The survival advantage for women, however, persists even after these factors have been taken into account. ${ }^{67}$ Women's better prognosis than men might therefore be interpreted as support for the argument that oestrogens inhibit the growth of melanoma, and recent studies supports this. In particular, endogenous oestrogens have an inhibitory effect on melanoma. ${ }^{48}$ Several cases have been reported of melanoma diagnosed in premenopausal women metastasising many years later in the postmenopausal period. ${ }^{8}$ In addition, five year survival in premenopausal women is significantly better than that in postmenopausal women. ${ }^{4}$

If endogenous oestrogens improve the outcome of melanoma then might exogenous oestrogens do so as well? The Melanoma Cooperative Group examined the relation between oestrogen administration and tumour characteristics in women with stage I melanoma. ${ }^{9}$ Women who had taken oral contraceptive at any time before diagnosis presented with significantly thinner tumours than women who hadn't, and women who used oral contraceptive in the year before melanoma was diagnosed had thinner lesions than those who had stopped use more than a year before. But oral contraceptives did not alter survival, probably because users had a higher incidence of truncal lesions than non users. A similar trend towards thinner lesions was also seen in those who used hormone replacement therapy.

The important question that arises from this work is, why do women who take oral contraceptives present with thinner lesions. It may not be a direct effect of oral contraceptives but could be due to oral contraceptive users seeking medical attention sooner.

Anecdotal reports have been published of rapid progression of melanoma during pregnancy. ${ }^{10}$ Melanomas associated with pregnancy are significantly thicker than melanomas in nonpregnant women, but the reason for this is unknown." When melanomas of identical thickness are compared there is no effect of pregnancy on survival, and pregnancy does not seem to increase the risks of recurrence after treatment for melanoma. ${ }^{12}$

Although many questions remain unanswered about the relation between melanoma and the hormonal environment, several conclusions are possible given the current state of knowledge. Firstly, there is no evidence that the use of exogenous oestrogens, either oral contraceptives or hormone replacement therapy, have a role in the aetiology of melanoma. Secondly, women have a survival advantage over men that could be due to an inhibitory effect of endogenous oestrogens on the growth of melanoma. Thirdly, exogenous oestrogens do not promote progression of the disease in patients with melanoma, so women who have previously been treated for melanoma can safely use hormonal supplements. And lastly, pregnancy seems to carry no adverse effect on survival after treatment for melanoma. Nevertheless, we advise patients with thick lesions $(>2 \mathrm{~mm}$ ) to consider delaying pregnancy 